\title{
Free-matrices min-projection control for high frequency DC-DC converters
}

\author{
Mathias Serieye, Carolina Albea-Sanchez and Alexandre Seuret
}

\begin{abstract}
This paper deals with the stabilization of high frequency DC-DC converters. This kind of systems can be modeled as switched affine systems subject to a periodic sampled-data control implementation. The dynamics of these systems is expressed using the $\delta$-operator in order to cope with high frequency switching control constraints. The novelties of this paper, first, relies on the formulation of a freematrices based min-projection control law, that allows the selection of the mode to be activated, based on the knowledge of the state variables. Second, the stability theorem, expressed in terms of a tractable optimization problem, that guarantees a practical stability result to a set and delivers the optimal control law that minimizes the volume of this one. The method is then illustrated through the control of a high frequency boost converter.
\end{abstract}

\section{INTRODUCTION}

Power converters are present in many systems such as electronic devices, power supplies, aircraft and automobile industries, photovoltaic installations, among others. This kind of systems needs to be managed by suitable control laws in order to increase their efficiency and reliability in their process of electric power transformation.

Often, power converters can be modeled as Switched Affine Systems (SAS's), which are a subclass of hybrid dynamical systems, i.e. they are composed of some functioning modes and a logical rule that selects the mode to be activated among the possible modes [10],[14],[15]. More precisely, the voltages and currents are continuoustime variables while the transistors, which allow the selection of mode, evolve at some discrete instants of time. A particularity of SAS's is that each mode may have its own equilibrium, which is, in general, not the same of the other. These equilibriums are solutions to the system in the generalized sense of Krasovskii. This characteristic renders this class of systems particularly interesting from the control point of view.

The literature on this power converters systems is quite rich and several control laws have been designed. One of the most popular approach relies on an average model, allowing to remove the hybrid nature of the systems and to focus on the study of a continuous-time model $[2],[17],[22]$. The price to pay is that the discrete behavior of the switches is lost, limiting the system performance. Another approach refers to the so-called Pulse Weight Modulator (PWM) approach [13],[20], which consists in controlling the ratio on the time spend in some modes over a hyper sampling period. Finally, several sliding mode control laws have been developed as for instance in $[16]$.

Last years, there have been remarkable efforts to analyze and control power converters by keeping their hybrid nature [4],[12],[23], often referring to the hybrid dynamical systems theory [7]. In this direction, one of the most popular approach consists in the introduction of minprojection control strategies [1],[5],[21]. These strategies consider aperiodic sampled-data control implementation. However, in some applications, it is required that the converters are periodically updated due to physical constraints [6], [13]. In this situation, it is not possible to guarantee the convergence of the trajectories to a given operating point, but to a neighborhood of it. Therefore, only practical stability results can be guaranteed, i.e. one can only ensure that the solutions to the system converge asymptotically to an invariant set, also denoted as an attractor. Therefore an objective is to design control laws that minimize the size the attractor in order to reduce the effects of the chattering around the equilibrium.

Some periodic sampled-data control laws that consider a min-projection strategy can be found in the literature. The reader may refer to [4], [10], [11], to get the general motivations. Interestingly, the authors of [5] present a Lyapunov matrix-based min-projection control law for discrete-time systems. They define an attractor through a level set of a quadratic Lyapunov function, that represents an ellipsoid whose center is allowed to be translated from the origin. The authors demonstrated therein that this shift has a notable impact when minimizing the size of attractor. However, this result is not suitable for high-frequency systems, as in the situation of power converters, due to numerical issues generated by a small sampling time. Alternatively, a relaxed controller is presented in [24], which does not depend on the Lyapunov matrix but is still structured by the system matrices. A model of high frequency DC-DC converters was considered in [24], using the $\delta$-operator, which was in introduced in [18]. The motivation for using such a modeling is to avoid computational issues, when using discrete-time model with a small sampling period [19]. Nevertheless, this work only provides the attractivity of a neighborhood of the equilibrium, which is not built with a level set of the Lyapunov function, which is centered at the equilibrium.

Inspired by [3], which concerns discrete-time switched affine systems, the objectives of this paper are summarized in the following items: 
- To design a free-matrices min-projection control, where the parameters of the control law are not structured by the Lyapunov matrix nor the systems matrices. The unstructured gain allows naturally to relax the resulting conditions and potentially to reduce the conservatism.

- To provide an accurate estimation of the attractor, whose center may be lightly shifted in order to minimize its volume.

- To derive conditions that guarantee the global asymptotic stability of the attractor for high frequency sampled-data control of SAS's using a dynamic model expressed using the $\delta$-operator.

- To apply this methodology to a class of DC-DC converters, as the boost converter.

The paper is organized as follows. Section II described the problem under consideration. Then, the proposed control law is presented in Section III. Section IV is devoted to the validation of our main result in simulations. The paper ends with a conclusion section.

Notations: Throughout the paper, $\mathbb{N}$ denotes the set of natural numbers, $\mathbb{R}$, the real numbers, $\mathbb{R}^{+}$, the positive real numbers, $\mathbb{R}^{n}$, the $n$-dimensional Euclidean space and $\mathbb{R}^{n \times m}$, the set of all real $n \times m$ matrices. The set composed by the first $N$ positive integers, namely $\{1,2, \ldots, N\}$, is denoted by $\mathbb{K}$. For any matrix $M$ of $\mathbb{R}^{n \times n}$, the notation $M \succ 0,(M \prec 0)$ means that $M$ is symmetric positive (negative) definite and $\operatorname{det}(M)$ represents its determinant. $\operatorname{He}(A)$ denotes the Hermitian matrix of $A$. Denote by $\Lambda_{\mathbb{K}} \subset \mathbb{R}^{N}$, the set defined by $\left\{\lambda \in[0,1]^{N} \mid \sum_{i \in \mathbb{K}} \lambda_{i}=1\right\}$.

\section{Problem formulation}

\section{A. Continuous-time model}

A DC-DC converter is a high-frequency switched affine system, modeled as follows [1], [4]

$$
\dot{z}=\mathbf{A}_{\sigma} z+\mathbf{b}_{\sigma},
$$

where $z \in \mathbb{R}^{n}$ is the state and gathers the voltages and currents of the converter. $\mathbf{A}_{\sigma}$ and $\mathbf{b}_{\sigma}$ are matrices of suited dimensions. Finally, the control input is $\sigma \in \mathbb{K}:=\{1,2 \ldots, N\}$, which represents the switching signal. This control signal may only be modified at time instants $t_{k}$, with $k \in \mathbb{N}$. In this paper, the length of the sampling interval $t_{k+1}-t_{k}=T$ between two successive switching instants is assumed to be constant, known and potentially small. The equilibrium set of (1) is defined as follows:

Definition 1: Consider the set $\Omega_{e}$ given by

$$
\Omega_{e}=\left\{z_{e} \in \mathbb{R}^{n}, \mathbf{A}_{\lambda} z_{e}+\mathbf{b}_{\lambda}=0, \quad \lambda \in \Lambda_{\mathbb{K}}\right\},
$$

where $\mathbf{A}_{\lambda}:=\sum_{i \in \mathbb{K}} \lambda_{i} \mathbf{A}_{i}$ and $\mathbf{b}_{\lambda}:=\sum_{i \in \mathbb{K}} \lambda_{i} \mathbf{b}_{i}$.

This definition encompasses some possible equilibriums. Indeed, it can exist other equilibriums outside of $\Omega_{e}$. On the other hand, we highlight that these periodic sampled-data systems are characterized by the fact that their trajectories do not necessarily converge into a given equilibrium $z_{e}$, but in a neighborhood of it.

Denoting $x:=z-z_{e}$, we get the error equation of (1),

$$
\dot{x}=\mathbf{A}_{\sigma} x+\mathcal{B}_{\sigma},
$$

where $\mathcal{B}_{\sigma}:=\mathbf{A}_{\sigma} z_{e}+\mathbf{b}_{\sigma}$ and the convex combination $\sum_{i \in \mathbb{K}} \lambda_{i} \mathcal{B}_{i}=0$ is verified for $\lambda \in \Lambda_{\mathbb{K}}$.

\section{B. Discrete-time model using the $\delta$-operator}

Following by [24], we propose in this paper a discretetime model based on the $\delta$-operator introduced by [18], which is appropriated for high-switching frequencies. Indeed, we can find in the literature a lot of interest in the use of the $\delta$-operator to avoid numerical issues in the computation of the discrete-time dynamics [18]. Let define the $\delta$-operator.

Definition 2 ([18]): For any function $\xi$ from $\mathbb{R}^{+}$to $\mathbb{R}^{n}$, let us define $\delta \xi_{k}$, at any time instant $t_{k} \in \mathbb{R}^{+}$, as follows

$$
\delta \xi_{k}:=\frac{1}{T}\left(\xi_{k+1}-\xi_{k}\right), \quad \forall k \geq 0,
$$

where we used the notations $\delta \xi_{k}=\delta \xi\left(t_{k}\right)$ and $\xi_{k}=\xi\left(t_{k}\right)$, for all $k \in \mathbb{N}$, to ease the readability.

Hence, we obtain the discrete-time dynamics of system (2) in the framework of the $\delta$-operator, which can be identified as a class of discrete switched affine systems

$$
\delta x_{k}=A_{\sigma} x_{k}+B_{\sigma},
$$

where $x_{k}=x\left(t_{k}\right)$ and where matrices $A_{\sigma}$ and $B_{\sigma}$ are given by

$$
\begin{aligned}
& A_{\sigma}=\frac{1}{T}\left(e^{\mathbf{A}_{\sigma} T}-I\right), \\
& B_{\sigma}=\frac{1}{T} \int_{0}^{T} e^{\mathbf{A}_{\sigma}(T-s)} d s \mathcal{B}_{\sigma}, \quad \sigma \in \mathbb{K} .
\end{aligned}
$$

This formulation presents an important characteristic compared to a common discrete-time formulation. Considering small values of $T$ in the usual discretization of the continuous-time equation (2) may lead to several numerical problems. However, when $T$ goes to zero, matrices $A_{\sigma}$ and $B_{\sigma}$ of the $\delta$-modulator model converge to $\mathbf{A}_{\sigma}$ and $\mathcal{B}_{\sigma}$, respectively, retrieving the continuoustime model (2). Another important issue is that matrices $A_{\sigma}$ and $B_{\sigma}$ depend explicitly on the switching period $T$.

Remark 1: The consideration of an equilibrium $z_{e}$ in $\Omega_{e}$, ensures that $\sum_{i \in \mathbb{K}} \lambda_{i} \mathcal{B}_{i}=0$. It is important to note that, in the sequel, we will not required the same constraint on the discretized model, i.e. there does not necessarily exist a linear combination of $\Lambda_{\mathbb{K}}$ such that $\sum_{i \in \mathbb{K}} \lambda_{i} B_{i}=0$. This represents an important relaxation of this paper with respect to the discrete-time formulation considered for instance in [3],[5].

Let us note that the continuous evolution of (1) during two sampling times is not encompassed in (3). Nevertheless, the continuous solution can be defined by integrating 
the solution over a sampling interval, leading, for all $t \in\left[t_{k}, t_{k}+T\right]$ and for all $k \in \mathbb{N}$, to

$$
x(t)=e^{\mathbf{A}_{\sigma}\left(t-t_{k}\right)} x\left(t_{k}\right)+\int_{t_{k}}^{t} e^{\mathbf{A}_{\sigma}\left(\tau-t_{k}\right)} d \tau \mathcal{B}_{\sigma} .
$$

Therefore, we can notice that the solutions to the system are bounded during the inter-sampling time.

As mentioned above, system (3) cannot be stabilized to the origin in general, but only to a neighborhood of it. Hence, the control problem have to be relaxed, considering attractor sets, which are not necessarily reduced to a point. In this paper, we aim at considering attractors that are expressed as a level set of a Lyapunov function given by

$$
V\left(x, x_{c}\right)=\left(x-x_{c}\right)^{T} P\left(x-x_{c}\right),
$$

where $\mathrm{P}$ is a positive definite matrix and $x_{c}$ is a vector to be defined, that allows to shift the center of the level set. Then, we define the attractor by

$$
\mathcal{A}:=\left\{x \in \mathbb{R}^{n} \text {, s.t. } V\left(x, x_{c}\right)<1\right\} .
$$

We are now in position to formulate the objectives of the paper.

Problem 1: The design of a switching control law $\sigma$ such that $\mathcal{A}$ is Uniformly Globally Asymptotically Stable (UGAS) for system (3). This can be summarized in the next three items:

(i) If $x_{k}$ is not in $\mathcal{A}$ at a given instant $k$, then $\delta V\left(x_{k}\right)$, defined by $\left(V\left(x_{k+1}\right)-V\left(x_{k}\right)\right) / T$, is definite negative.

(ii) The origin belongs to $\mathcal{A}$.

(iii) If $x_{k}$ is in $\mathcal{A}$ at a given time instant $k$, then $x_{k+1}$ also belongs to $\mathcal{A}$.

\section{Free Matrix-BASED SWITChing CONTROL DESIGN}

\section{A. Main result}

Compared to [24] where the authors use a minprojection control law structured in the system matrices $A_{\sigma}$ and $B_{\sigma}$, we propose here a free-matrices minprojection control law. This is formalized in the following theorem

Theorem 1: For a given $z_{e} \in \Omega_{e}$, assume that matrices $P \in \mathbb{R}^{n \times n} \succ 0, h \in \mathbb{R}^{n}$, and $N_{i}=N_{i}^{T} \in \mathbb{R}^{(n+1) \times(n+1)}$ as well as a parameter $\mu \in(0,1)$ are the solutions to the optimization problem

$$
\begin{aligned}
& \min _{\mu, P, h, N_{i}} \quad-\log (\operatorname{det}(P)) \\
& \text { s.t. } \quad P \succ 0, \\
& {\left[\begin{array}{rr}
\Psi_{i}+N_{\lambda}-N_{i}-\frac{\mu}{T}\left[\begin{array}{ll}
0 & 0 \\
* 1
\end{array}\right] & \frac{\mu}{T}\left[\begin{array}{c}
P \\
h^{\top}
\end{array}\right] \\
* & -\frac{\mu}{T} P
\end{array}\right] \prec 0, \quad \forall i \in \mathbb{K},} \\
& \operatorname{He}\left(h^{\top} B_{\lambda}\right)+T \sum_{i \in \mathbb{K}} \lambda_{i} B_{i}^{\top} P B_{i} \succ 0,
\end{aligned}
$$

where

$$
\begin{aligned}
\Psi_{i} & :=\operatorname{He}\left(\left[\begin{array}{l}
A_{i}^{\top} \\
B_{i}^{\top}
\end{array}\right][P h]\right)+T\left[\begin{array}{l}
A_{i}^{\top} \\
B_{i}^{\top}
\end{array}\right] P\left[A_{i} B_{i}\right], \\
N_{\lambda} & :=\sum \lambda_{i} N_{i} .
\end{aligned}
$$

Then, the switching control law given by

$$
\sigma\left(x_{k}\right) \in \underset{i \in \mathbb{K}}{\operatorname{argmin}}\left[\begin{array}{c}
x_{k} \\
1
\end{array}\right]^{T} N_{i}\left[\begin{array}{c}
x_{k} \\
1
\end{array}\right]
$$

ensures that $\mathcal{A}$ is UGAS for system (3) and of a minimum size.

For the sake of simplicity, the control law $\sigma\left(x_{k}\right)$ will be denoted simply as $\sigma$ when there is no confusion.

Remark 2: By selecting parameter $\mu$ in $(0,1)$, the nonconvex optimization problem (7)-(10) can be reformulated as a Linear Matrix Inequality (LMI), and consequently into a convex problem. Then, a line-search routine on $\mu \in(0,1)$ allows deriving the optimum of the problem, as performed in [5].

Remark 3: The optimization problem aims at maximizing $\log (\operatorname{det}(P))$ as in [5]. This problem can be interpreted as the minimization of the volume of the ellipsoid defined by $\mathcal{A}$.

Proof: To prove this theorem, we have to ensure that the conditions therein satisfy the three items. Assume that there exist a positive definite matrix $P$, a vector $h$ and symmetric matrices $N_{i}$, for $i \in \mathbb{K}$, that are solution to the convex problem (8)-(10) for a given positive parameter $\mu \in(0,1)$.

Proof of (i) : Let us assume that the state $x_{k}$ does not belong to $\mathcal{A}$. Then, by selecting $x_{c}=-P^{-1} h$ in the Lyapunov function given in (5) we have :

$$
V\left(x_{k},-P^{-1} h\right)=\left(x_{k}+P^{-1} h\right)^{\top} P\left(x_{k}+P^{-1} h\right)>1 .
$$

The positive definiteness of $V$ is guaranteed by the assumption on the positive definiteness of matrix $P$. The computation of $\delta V\left(x_{k}\right)$ is given by

$$
T \delta V\left(x_{k},-P^{-1} h\right)=V\left(x_{k+1},-P^{-1} h\right)-V\left(x_{k},-P^{-1} h\right) .
$$

Replacing $x_{k+1}$ by $x_{k}+T \delta x_{k}$ in the expression of $V\left(x_{k+1},-P^{-1} h\right)$ leads to

$$
\delta V\left(x_{k},-P^{-1} h\right)=T \delta x_{k}^{\top} P \delta x_{k}+2 \delta x_{k}^{\top} P\left(x_{k}+P^{-1} h\right) .
$$

Then, $\delta x_{k}$ is replaced by its expression in (3) and the following expression of $\delta V$ is derived

$$
\begin{aligned}
\delta V\left(x_{k},-P^{-1} h\right) & =\left[\begin{array}{c}
x_{k} \\
1
\end{array}\right]^{\top}\left\{T\left[\begin{array}{c}
A_{\sigma}^{\top} \\
B_{\sigma}^{\top}
\end{array}\right] P\left[A_{\sigma} B_{\sigma}\right]\right. \\
& \left.+\operatorname{He}\left(\left[\begin{array}{c}
A_{\sigma}^{\top} \\
B_{\sigma}^{\top}
\end{array}\right]\left[\begin{array}{ll}
P & h
\end{array}\right]\right)\right\}\left[\begin{array}{c}
x_{k} \\
1
\end{array}\right],
\end{aligned}
$$

or, equivalently,

$$
\delta V\left(x_{k},-P^{-1} h\right)=\left[\begin{array}{c}
x_{k} \\
1
\end{array}\right]^{\top} \Psi_{\sigma}\left[\begin{array}{c}
x_{k} \\
1
\end{array}\right],
$$

where $\sigma \in \mathbb{K}$ corresponds to the switching signal at time $t_{k}$ and where $\Psi_{\sigma}$ is defined in (11). 
The next two steps aim at including the information that mode $\sigma$ is the actual control mode and also that the state $x_{k}$ is assumed to be outside of $\mathcal{A}$. To do so, note that, if $\sigma$ results from the application of the switching control law (13), when the state is at $x_{k}$, we have

$$
\left[\begin{array}{c}
x_{k} \\
1
\end{array}\right]^{\top}\left(N_{j}-N_{\sigma}\right)\left[\begin{array}{c}
x_{k} \\
1
\end{array}\right] \geq 0, \quad \forall j \in \mathbb{K} .
$$

Therefore, for any convex combination $\lambda \in \Lambda_{\mathbb{K}}$, and more especially for the particular case $\lambda$ corresponding to Definition 1 , we have

$$
\sum_{j \in \mathbb{K}} \lambda_{j}\left(N_{j}-N_{\sigma}\right),=N_{\lambda}-N_{\sigma}
$$

that, together with the previous inequality, yields

$\sum_{j \in \mathbb{K}} \lambda_{j}\left[\begin{array}{c}x_{k} \\ 1\end{array}\right]^{\top}\left(N_{j}-N_{\sigma}\right)\left[\begin{array}{c}x_{k} \\ 1\end{array}\right]=\left[\begin{array}{c}x_{k} \\ 1\end{array}\right]^{\top}\left(N_{\lambda}-N_{\sigma}\right)\left[\begin{array}{c}x_{k} \\ 1\end{array}\right] \geq 0$

which, again, reflects that, for this state value $x_{k}$, mode $\sigma$ is one of the possible active mode. In a second step, we notice that the negative definiteness of $\delta V$ is only required outside $\mathcal{A}$, i.e. for any $x \in \mathbb{R}^{n}$, such that $V\left(x,-P^{-1} h\right)>1$ and can be easily rewritten as follows

$$
\forall x \notin \mathcal{A}, \quad\left[\begin{array}{l}
x \\
1
\end{array}\right]^{\top}\left[\begin{array}{cc}
P & h \\
h^{\top} & h^{\top} P^{-1} h-1
\end{array}\right]\left[\begin{array}{l}
x \\
1
\end{array}\right]>0
$$

Hence, if for $\lambda \in \Lambda_{\mathbb{K}}$, there exist matrices $P, h$ and $N_{i}$, for $i \in \mathbb{K}$, such that condition

$$
\left[\begin{array}{c}
x_{k} \\
1
\end{array}\right]^{\top} \Psi_{\sigma}\left[\begin{array}{c}
x_{k} \\
1
\end{array}\right]<0
$$

holds for all $\left(x_{k}, \sigma\left(x_{k}\right)\right) \in \mathbb{R}^{n} \times \mathbb{K}$ that verify

$$
\begin{aligned}
& {\left[\begin{array}{c}
x_{k} \\
1
\end{array}\right]^{\top}\left[\begin{array}{cc}
P & h \\
h^{\top} & h^{\top} P^{-1} h-1
\end{array}\right]\left[\begin{array}{c}
x_{k} \\
1
\end{array}\right]>0 } \\
& \text { and }\left[\begin{array}{c}
x_{k} \\
1
\end{array}\right]^{\top}\left(N_{\lambda}-N_{\sigma}\right)\left[\begin{array}{c}
x_{k} \\
1
\end{array}\right] \geq 0,
\end{aligned}
$$

then, (i) holds. This problem can be recast, using Sprocedures, into the existence of a positive scalar $\mu>0$, which yields to the following inequality:

$$
\Psi_{\sigma}+N_{\lambda}-N_{\sigma}+\frac{\mu}{T}\left[\begin{array}{cc}
P & h \\
h^{\top} & h^{\top} P^{-1} h-1
\end{array}\right] \prec 0, \quad \sigma \in \mathbb{K} .
$$

Note that, in the previous expression, the tuning parameter $\mu$ is divided by the sampling period $T$, in order to simplify the conditions latter on. Lastly, by noting that

$$
\left[\begin{array}{cc}
P & h \\
h^{\top} & h^{\top} P^{-1} h
\end{array}\right]=\left[\begin{array}{c}
P \\
h^{\top}
\end{array}\right] P^{-1}\left[\begin{array}{c}
P \\
h^{\top}
\end{array}\right]^{\top},
$$

the application of the Schur complement to this matrix in equation (17) allows us to retrieve condition (9) and to conclude the proof of item (i).
Proof of (ii): The objective is here to prove that the $\overline{\text { equilibrium } x}=0$ belongs to $\mathcal{A}$, i.e.

$$
V\left(0,-P^{-1} h\right)=h^{\top} P h<1 .
$$

To do this, let us compute the linear combination of (17), weighted by $\lambda$. This yields

$$
\sum_{i \in \mathbb{K}} \lambda_{i}\left(\Psi_{i}+N_{\lambda}-N_{i}+\frac{\mu}{T}\left[\begin{array}{cc}
P & h \\
h^{\top} & h^{\top} P^{-1} h-1
\end{array}\right]\right),
$$

which is guaranteed to be negative definite from LMI conditions. By using $\sum_{i \in \mathbb{K}} \lambda_{i}=1$ and the fact that $\sum_{i \in \mathbb{K}} \lambda_{i} N_{i}=N_{\lambda}$, the previous inequality reduces to

$$
\sum_{i \in \mathbb{K}} \lambda_{i} \Psi_{i}+\frac{\mu}{T}\left[\begin{array}{cc}
P & h \\
h^{\top} & h^{\top} P^{-1} h-1
\end{array}\right] \prec 0 .
$$

The idea is to extract from this inequality a necessary condition leading to the condition $h^{\top} P^{-1} h \leq 1$. Indeed, the coefficient of the matrix located at position $(n+1, n+$ 1 ) is necessarily negative definite. An expression of the coefficient is obtained by expanding the $\Psi_{i}$ 's matrices, which leads to

$$
\operatorname{He}\left(h^{\top} B_{\lambda}\right)+\sum_{i \in \mathbb{K}} \lambda_{i} T B_{i}^{\top} P B_{i}+\frac{\mu}{T}\left(h^{\top} P^{-1} h-1\right)<0 .
$$

According to inequality (10), we have that

$$
\begin{aligned}
& h^{\top} P^{-1} h-1<h^{\top} P^{-1} h-1 \\
& \quad+\frac{T}{\mu}\left[\operatorname{He}\left(h^{\top} B_{\lambda}\right)+\sum_{i \in \mathbb{K}} \lambda_{i} T B_{i}^{\top} P B_{i}\right]<0 .
\end{aligned}
$$

Hence, a necessary condition for (9) to hold is that $h^{\top} P^{-1} h-1<0$, which corresponds exactly to the condition $V\left(0,-P^{-1} h\right)<1$, concluding the proof of (ii).

Proof of (iii) Let us now assume that the state $x_{k}$ at a given time instant $k$ is inside $\mathcal{A}$, i.e. $V\left(x_{k},-P^{-1} h\right)<1$. We have seen from the definition of the $\delta$ operator and from the proof of item (i), that the following expression of $V\left(x_{k+1},-P^{-1} h\right)$ holds,

$$
\begin{aligned}
V\left(x_{k+1},-P^{-1} h\right) & =V\left(x_{k},-P^{-1} h\right)+T \delta V\left(x_{k},-P^{-1} h\right) \\
& =V\left(x_{k},-P^{-1} h\right)+T\left[\begin{array}{c}
x_{k} \\
1
\end{array}\right]^{\top} \quad \Psi_{\sigma}\left[\begin{array}{c}
x_{k} \\
1
\end{array}\right]
\end{aligned}
$$

From (9), an upper bound of $V\left(x_{k+1},-P^{-1} h\right)$ is obtained as follows

$$
\begin{aligned}
& V\left(x_{k+1},-P^{-1} h\right)< V\left(x_{k},-P^{-1} h\right) \\
&-\mu\left[\begin{array}{c}
x_{k} \\
1
\end{array}\right]^{\top}\left[\begin{array}{cc}
P & h \\
h^{\top} & h^{\top} P^{-1} h-1
\end{array}\right]\left[\begin{array}{c}
x_{k} \\
1
\end{array}\right] \\
&-T\left[\begin{array}{c}
x_{k} \\
1
\end{array}\right]^{\top}\left(N_{\lambda}-N_{\sigma}\right)\left[\begin{array}{c}
x_{k} \\
1
\end{array}\right], \\
&=(1-\mu) V\left(x_{k},-P^{-1} h\right)+\mu \\
&-T\left[\begin{array}{c}
x_{k} \\
1
\end{array}\right]^{\top}\left(N_{\lambda}-N_{\sigma}\right)\left[\begin{array}{c}
x_{k} \\
1
\end{array}\right],
\end{aligned}
$$

where $\sigma$ refers to the active mode at this time instant imposed by control law (13). This last expression has been 


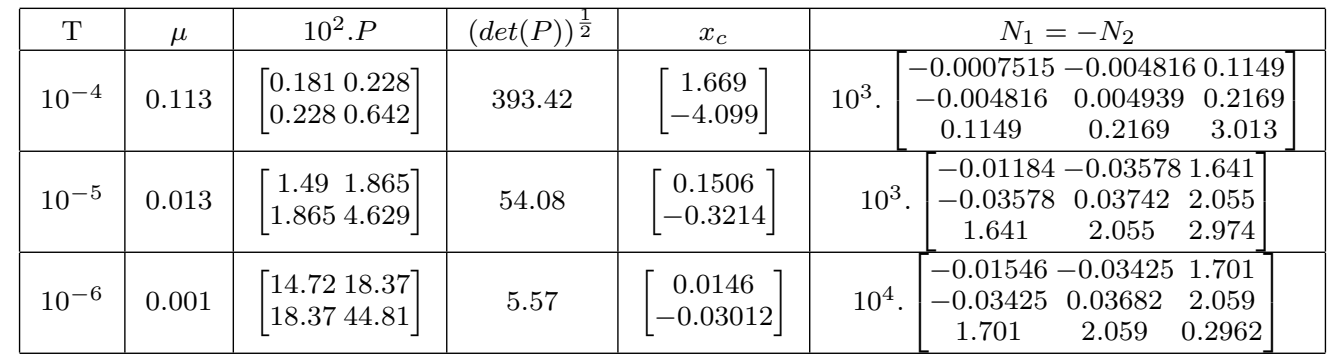

TABLE I: Numerical solutions obtained by solving Theorem 1, for different values of the sampling period $T$.

obtained by recognizing the expression of the Lyapunov function in the second term. Together with inequality (16), we are able to ensure that

$$
V\left(x_{k+1},-P^{-1} h\right) \leq(1-\mu) V\left(x_{k},-P^{-1} h\right)+\mu .
$$

Since $x_{k}$ is assumed in $\mathcal{A}$, we have $V\left(x_{k},-P^{-1} h\right) \leq 1$. Moreover the assumption that $\mu$ is in $(0,1)$, then $1-\mu$ is positive, so that we have

$$
V\left(x_{k+1},-P^{-1} h\right) \leq(1-\mu)+\mu=1 .
$$

This proves that $x_{k+1}$ also belongs to $\mathcal{A}$, which concludes the proof.

\section{B. Comments on the main result}

As mentioned in Remark 1, the previous theorem does not require that there exists a linear combination $\lambda^{\prime}$ in $\Lambda_{\mathbb{K}}$ such that $B_{\lambda^{\prime}}$ is zero. From the practical point of view, DC-DC converter models are originally provided in continuous-time and, in most case, the equilibrium in Definition 1 are defined in continuous time, and there is no guarantee that the same formulation also holds in discrete-time (unless matrices $A_{i}$ are the same). The price to pay is the introduction of a new condition in the optimization problem, referring to as inequality (10). It is however easy to see that, if there exists a linear combination $\lambda^{\prime}$ in $\Lambda_{\mathbb{K}}$ such that $B_{\lambda^{\prime}}=0$, then inequality (10) follows straightforwardly.

Apart from the computational benefits for high frequency, the $\delta$-operator approach has the particularity to have continuity of the LMI conditions when the sampling period $T$ tends to 0 . Indeed, it is easy to extract from condition (9) the stability condition that would be obtained for the continuous-time version of the problem.

\section{Applichtion to a Boost converter}

This section aims at illustrating how Theorem 1 is applicable to the control of a classical boost converter, characterized by two functioning modes and a switching control working at high frequency. This system is governed by (1) where $z:=\left[\begin{array}{ll}i_{L} & v_{C}\end{array}\right]^{\top}$, where $i_{L}$ is the inductor current, and $v_{C}$, the capacitor voltage. The matrices that define the modes are as follows

$$
\mathbf{A}_{\sigma}=\left[\begin{array}{cc}
-\frac{R}{L} & \frac{(1-\sigma)}{L} \\
\frac{(\sigma-1)}{C_{o}} & -\frac{1}{R_{o} C_{o}}
\end{array}\right], \quad \mathbf{b}_{\sigma}=\left[\begin{array}{c}
\frac{V_{i n}}{L} \\
0
\end{array}\right] .
$$

The parameters are given by $V_{i n}=100 \mathrm{~V}, R=2 \Omega$, $L=500 \mu \mathrm{H}, C_{o}=470 \mu \mathrm{F}$ and $R_{o}=50 \Omega$. Finally, the chosen equilibrium point is $z_{e}=\left[\begin{array}{c}3 \\ 120\end{array}\right] \in \Omega_{e}$ with $\lambda=$ [ 0.220 .78$]$. The optimization problem in Theorem 1 is solved in Matlab by using sedumi and the CVX solver [8], [9] together with a line-search routine that selects the parameter $\mu$, as mentioned in Remark 2. Table I gathers the matrices and parameters obtained for the switching time, $T=3.10^{-5}, 10^{-5}, 10^{-6}$. As expected, decreasing $T$ leads to a reduction for the volume of $\mathcal{A}$, and as a consequence, the center of the ellipsoid is closer to $z_{e}$.

Figure 1 depicts several simulations that are obtained for the different values of $T$. A zoom of switching signal, marked by a grey patch in the first column. This figure nicely illustrates the practical stability result presented in this paper. Indeed, one can see the chattering that occurs around the equilibrium and that their amplitude increases as $T$ grows. The third column of Figure 1, shows the evolution of the Lyapunov function and its position with respect to 1 , that specifies if the state enters $\mathcal{A}$. This allows verifying that, once the trajectory enters $\mathcal{A}$, it never leaves. Finally, we perform in the last column the state evolution in the state plane and $\mathcal{A}$. We highlight as $\mathcal{A}$ is reduced as $T$ diminishes.

\section{Conclusions}

In this paper, we have proposed a min-projection control for power converters working at high frequency, as required in practical aspects of these applications. The novelty of this paper relies on the introduction of a freematrices min-projection control law. The simple control structure allows to have a lower computational load with respect to the classical Lyapunov min-projection control. The use of the $\delta$-operator to model high frequency converters appears particularly relevant for this application. Moreover, the features help characterizing the set $\mathcal{A}$, that captures the chattering effects around the equilibrium.

According to us, there are many perspectives to this contribution. Among them, the question of the robustness with respect to parameter uncertainties or variations or jitter in the sampling period are of high interests for practical purposes. 

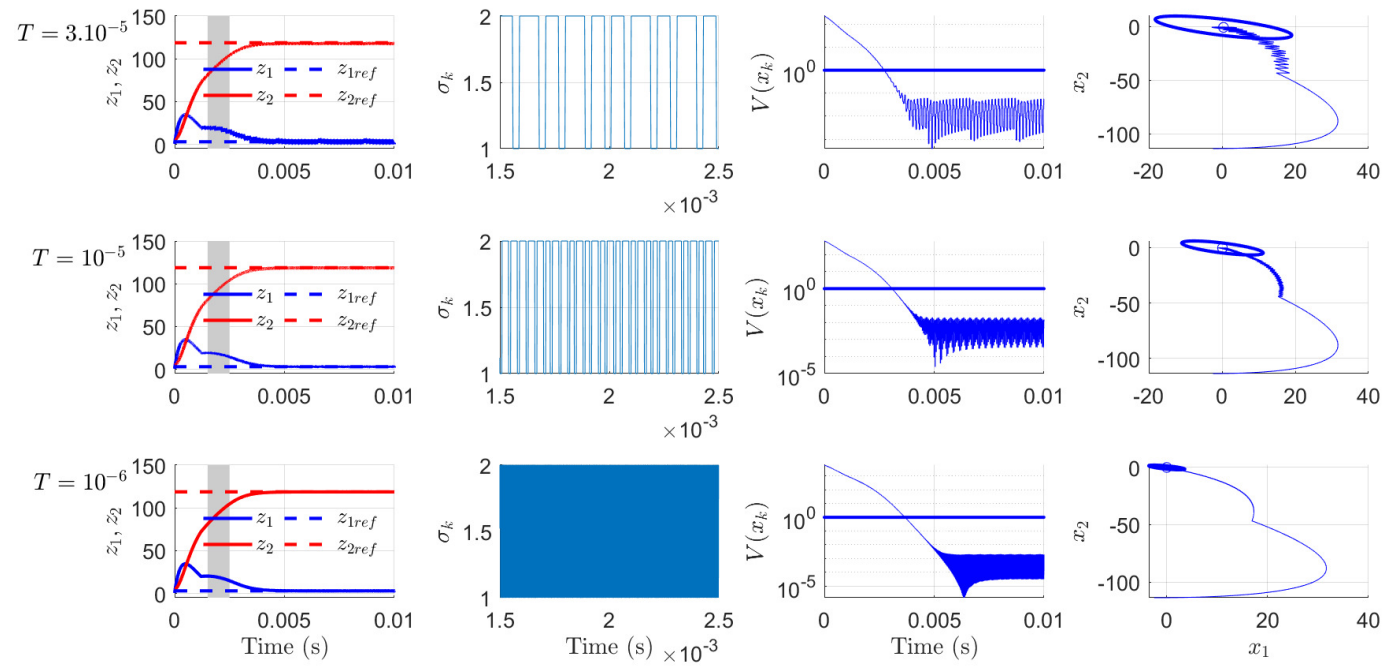

Fig. 1: Simulation results of switched affine system (1) with matrices (19) . From top to bottom, the different rows correspond to simulations results with sampling period $T=3.10^{-5}, 10^{-5}$ and $10^{-6}$ sec. From left to right, the different plots show the evolution of the state variables $\left(z_{1}, z_{2}\right)$, the switching signal $\sigma$ and the Lyapunov function $V\left(x_{k},-P^{-1}\right)$, with respect to time. The last column shows the trajectories of the error variable $x_{k}$ in the phase portrait.

\section{ACKNOWLEDGMENTS}

This work has been partially funded under grant "HISPALIS" ANR-18-CE40-0022-01.

\section{REFERENCES}

[1] C. Albea, G. Garcia, and L. Zaccarian. Hybrid dynamic modeling and control of switched affine systems: application to DC-DC converters. In IEEE Conference on Decision and Control, Osaka, Japan, 2015.

[2] C. Albea, F. Gordillo, and C. Canudas-de Wit. Adaptive control design for a boost inverter. Control Engineering Practice, 19(1):32-44, 2011.

[3] C. Albea Sanchez, A. Ventosa-Cutillas, A. Seuret, and F. Gordillo. Robust switching control design for uncertain discrete-time switched affine systems. Rapport LAAS n.18307, December 2018.

[4] G. S. Deaecto, J. C. Geromel, F.S. Garcia, and J.A. Pomilio. Switched affine systems control design with application to DC-DC converters. IET Control Theory \& Applications, 4(7):1201-1210, 2010.

[5] G.S. Deaecto and J.C. Geromel. Stability analysis and control design of discrete-time switched affine systems. IEEE Trans. on Automatic Control, 62(8):4058-4065, 2017.

[6] R.W. Erickson and D. Maksimovic. Fundamentals of power electronics. Springer Science \& Business Media, 2007.

[7] R. Goebel, R.G. Sanfelice, and A.R. Teel. Hybrid Dynamical Systems: modeling, stability, and robustness. Princeton University Press, 2012.

[8] M. Grant and S. Boyd. Graph implementations for nonsmooth convex programs. In V. Blondel, S. Boyd, and H. Kimura, editors, Recent Advances in Learning and Control, Lecture Notes in Control and Information Sciences, pages 95-110. Springer-Verlag Limited, 2008.

[9] M. Grant and S. Boyd. CVX: Matlab software for disciplined convex programming, version 2.1, mar 2014.

[10] P. Hauroigne, P. Riedinger, and C. Iung. Switched affine systems using sampled-data controllers: Robust and guaranteed stabilisation. IEEE Trans. on Automatic Control, 56(12):2929-2935, 2011.
[11] L. Hetel and E. Bernuau. Local stabilization of switched affine systems. IEEE Trans. on Automatic Control, 60(4):1158$1163,2015$.

[12] L. Hetel and E. Fridman. Robust sampled-data control of switched affine systems. IEEE Trans. on Automatic Control, 58(11):2922-2928, 2013.

[13] M.M.R Khan and W. Zhi-Ming. A generalized framework for sampled-data model analysis of closed-loop and PWM DC-DC converter system. In Proc. of the IEEE Industrial Electronics Society Conference (IECON), volume 2, pages 820-825, 2001.

[14] D. Liberzon. Switching in systems and control. Springer Science \& Business Media, 2003.

[15] D. Liberzon and A.S. Morse. Basic problems in stability and design of switched systems. IEEE Control Systems Magazine, 19(5):59-70, 1999.

[16] L. Martínez-Salamero, G. Garcia, M. Orellana, C. Lahore, B. Estibals, C. Alonso, and C.E. Carrejo. Analysis and design of a sliding-mode strategy for start-up control and voltage regulation in a buck converter. IET Power Electronics, 6(1):52-59, 2013.

[17] R.D Middlebrook and S. Cuk. A general unified approach to modelling switching-converter power stages. In IEEE Power Electronics Specialists Conference, pages 18-34, 1976.

[18] R.H. Middleton and G.C. Goodwin. Improved Finite Word Length Characteristics in Digital Control Using Delta Operators. IEEE Trans. on Automatic Control, 31(11):1015-1021, 1986.

[19] R.H. Middleton and G.C. Goodwin. Digital Control and Estimation: A Unified Approach. Prentice Hall, 1990.

[20] C. Olalla, R. Leyva, A. El Aroudi, P. Garces, and I. Queinnec. LMI robust control design for boost PWM converters. IET Power Electronics, 3(1):75-85, 2010.

[21] S. Pettersson and B. Lennartson. Stabilization of hybrid systems using a min-projection strategy. In Proc. of the 2001 IEEE American Control Conference, 2001., volume 1, pages 223-228.

[22] H. Sira-Ramírez and R. Silva-Ortigoza. Control design techniques in power electronics devices. Springer Science \& Business Media, 2006.

[23] T. A.F. Theunisse, J. Chai, R.G. Sanfelice, and W.P. Heemels. Robust global stabilization of the DC-DC boost converter 
via hybrid control. IEEE Trans. on Circuits and Systems I: Regular Papers, 62(4):1052-1061, 2015.

[24] A. Ventosa-Cutillas, C. Albea, A. Seuret, and F. Gordillo. Relaxed periodic switching controllers of high-frequency DCDC converters using the $\delta$-operator formulation. In 2018 IEEE Conference on Decision and Control (CDC), pages 3433-3438. IEEE, 2018. 